\title{
Backbone assignment of crystalline E. coli maltose binding protein Tobias Schubeis ${ }^{a}$, Jan Stanek ${ }^{b}$ and Guido Pintacuda ${ }^{a}$
}

\author{
a Université de Lyon, Centre de Résonance Magnétique Nucléaire à Très Hauts champs (UMR 5082 - \\ CNRS / Ecole Normale Supérieure de Lyon / Université Claude Bernard Lyon 1), 5 rue de la Doua, 69100 \\ Villeurbanne (France) \\ ${ }^{b}$ University of Warsaw, Faculty of Chemistry, Żwirki i Wigury 101, 02089 Warsaw, Poland
}

\section{Abstract}

The E.coli maltose binding protein (MBP) is a $42.5 \mathrm{kDa}$ molecule widely employed in many biotechnology applications. Because of its molecular size, it has become the main model system for the development of solution NMR methods adapted to large biomolecular targets. Here, we report virtually complete ( 90\%) backbone resonance assignments obtained on a microcrystalline sample of MBP with ${ }^{1} \mathrm{H}$-detected solidstate NMR at fast (>100 kHz) magic-angle spinning. We additionally present the detailed description of the methodology employed for the preparation of the sample and the acquisition and analysis of the NMR spectra. The chemical shifts, obtained with a single uniformly ${ }^{15} \mathrm{~N},{ }^{13} \mathrm{C}$-labelled and fully-protonated sample and about two weeks on a $800 \mathrm{MHz}$ NMR spectrometer, have been deposited to the BMRB under the accession number 50089 .

\section{Introduction}

The Escherichia coli protein MalE, commonly known as maltose binding protein (MBP), is responsible for maltose uptake (Boos and Shuman 1998) and consists of two globular domains that are connected by three linker segments. X-ray crystallography structures of the free (apo) or ligand-bound (holo) protein have revealed a $35^{\circ}$ angle between the two domains (Sharff et al. 1992). Domain motions and conformational equilibria have been extensively studied by solution NMR and other biophysical methods such as EPR and single-molecule FRET (Gardner 1998; Seo et al. 2014; Selmke et al. 2018). Using paramagnetic relaxation enhancements (PRE), it was shown that the holo state maintains in solution its crystallographic structure. In absence of ligands, MBP was found to populate a minor partially closed state in equilibrium with the major open structure (Tang et al. 2007).

MBP is a popular protein expression tag and a powerful crystallization mediator of target proteins (Waugh 2016). It is also frequently used for solution NMR method developments, thanks to its high solubility, thermostability and excellent chemical shift dispersion in two-dimensional correlation spectra (Yang and Kay 1999; Madl et al. 2009; Takeuchi et al. 2015). Nevertheless, due to slow tumbling, resonance assignment of a macromolecule of such size $(42.5 \mathrm{kDa})$ requires extensive deuteration to ensure sensitivity and resolution in triple-resonance spectra.

Recent leaps forward in probe technology allow today the acquisition of proton-detected solid-state NMR with high resolution and sensitivity. This approach does not require deuteration, and relies on simplified labelling $\left({ }^{13} \mathrm{C},{ }^{15} \mathrm{~N}\right)$ and only sub-milligram protein quantities (Mandala and Hong 2019). Since coherence lifetimes and resonance linewidths (and thus resolution) under MAS does not depend on the molecular weight of the target, solid-state NMR has already established itself as a highly complementary method in integrated structural biology, especially with respect to investigations of dynamics and interactions of proteins of considerable size. Nevertheless, spectral congestion and peak intensities are dramatically affected by an increase in protein size. With $>350$ residues and ${ }^{1} \mathrm{H}$ linewidths of about $100 \mathrm{~Hz}$ at $\sim 100 \mathrm{kHz}$ MAS, MBP still poses a considerable challenge to the current state-of-the-art solid-state NMR methodology, and calls for improvements in resolution, sensitivity, as well as more efficient methods of analysis. 
We have recently presented a new approach referred to as RAVASSA ("Redundant Assignment Via A Single Simultaneous Acquisition"), to simultaneously acquire amide-proton and alpha-proton-detected spectra (Stanek et al. 2020). This method provides significant time savings in data acquisition and at the same time accelerates data analysis. It notably improves the consistency between the position of a given resonance in different datasets, allowing sequential resonance linking thanks to automatic routines with high performance and reliability. Automated data evaluation provided by FLYA resulted in the assignment of resonances from $82 \%$ of the backbone residues in a crystalline sample of fully-protonated MBP.

Here we report an improved chemical shift assignment after manual evaluation and analysis of additional correlation spectra. We believe that the resonance assignments obtained in the solid state, together with the detailed description of the protocols for sample preparation and data acquisition and analysis, would be of great interest for the ensemble of NMR developers and users, given the role that MBP occupies as a model system for pushing the current size limits and opening new pathways towards the routine analysis of larger proteins. We additionally present a comparison of the results obtained with chemical shifts previously obtained on deuterated MBP in solution (Evenäs et al. 2001).

Considering the recent findings of previously unobserved dynamics in other, supposedly rigid, crystalline proteins (Rovó et al. 2019; Bonaccorsi et al. 2020), we additionally anticipate the possibility of new insight on internal motions which escaped previous determinations by X-ray and solution NMR.

\section{Methods and experiments}

Sample preparation

MBP (MalE 27-396, UniProt - POAEX9) was expressed in Escherichia coli cultures and purified using standard affinity and ion-exchange chromatography methods. Uniform ${ }^{13} \mathrm{C}$ and ${ }^{15} \mathrm{~N}$ isotope labeling was introduced by use of a minimal medium containing $3 \mathrm{~g} / \mathrm{l} \mathrm{U}-13 \mathrm{C}$-glucose and $1 \mathrm{~g} / \mathrm{l} 15 \mathrm{~N}$-ammonium chloride (Eurisotop). Eventually MBP was dialyzed against $10 \mathrm{mM}$ HEPES, $1 \mathrm{mM}$ Maltose $\mathrm{pH} 7.5$ and concentrated to $5 \mathrm{mg} / \mathrm{ml}$. Crystals were grown by sitting-drop vapor diffusion in 24-well plates sealed with a clear tape (Hampton Research). Initial screening was performed using $2 \mu$ protein drops $+2 \mu \mathrm{l}$ reservoir solution. For the NMR samples, the drops were composed of $20 \mu \mathrm{l}$ protein and reservoir solution, placed above $800 \mu \mathrm{l}$ reservoir in the well. The optimized reservoir solutions were $10 \mathrm{mM}$ HEPES $\mathrm{pH} 7.5,3 \mathrm{M}$ ammonium sulfate (AS) and 25\% PEG 8000, $100 \mathrm{mM}$ HEPES pH 7.2, $100 \mathrm{mM} \mathrm{MgCl}_{2}$. Crystals grew at room temperature over several days. NMR samples were packed into $0.7 \mathrm{~mm}$ MAS rotors by centrifugation at $100,000 \cdot \mathrm{g}$ at $10^{\circ} \mathrm{C}$ using a $1.3 \mathrm{~mm}$ ultracentrifuge tool (Giotto Biotech).

NMR spectroscopy

Experiments were performed on Bruker AVANCE III spectrometers with $\mathrm{B}_{0}$ of 18.8 or $23.5 \mathrm{~T}\left({ }^{1} \mathrm{H}\right.$ frequencies of 800 or $1 \mathrm{GHz}$, respectively) using triple-resonance $0.7 \mathrm{~mm}$ MAS probes. The sample rotation frequency was maintained at $107 \mathrm{kHz}$, and the sample temperature was approximately $15^{\circ} \mathrm{C}$. An experiment with simultaneous acquisition (Stanek et al. 2020) recorded at $\omega\left({ }^{1} \mathrm{H}\right) / 2 \pi=800 \mathrm{MHz}$ in 4.8 days resulted in a set of six three dimensional spectra: $(\mathrm{H}) \mathrm{CANH},(\mathrm{H}) \mathrm{CA}(\mathrm{CO}) \mathrm{NH},(\mathrm{H}) \mathrm{NCAHA},(\mathrm{H}) \mathrm{N}(\mathrm{CO}) \mathrm{CAHA},(\mathrm{H}) \mathrm{N}(\mathrm{CACO}) \mathrm{NH}$, $(\mathrm{H}) \mathrm{CA}(\mathrm{NCO}) \mathrm{CAHA}$. The set was complemented with conventionally recorded $(\mathrm{HCA}) \mathrm{CBCAHA}(800 \mathrm{MHz}$, $17 \mathrm{~h}),(\mathrm{HCA}) \mathrm{CB}(\mathrm{CA}) \mathrm{NH}(1 \mathrm{GHz}, 26 \mathrm{~h}),(\mathrm{H}) \mathrm{CONH}(800 \mathrm{MHz}, 1.5 \mathrm{~d}),(\mathrm{H}) \mathrm{CO}(\mathrm{CA}) \mathrm{NH}(800 \mathrm{MHz} 2.5 \mathrm{~d})$, $(\mathrm{H}) \mathrm{COCAHA}(800 \mathrm{MHz} 1.5 \mathrm{~d})$ and $(\mathrm{H}) \mathrm{CO}(\mathrm{N}) \mathrm{CAHA}(800 \mathrm{MHz}, 2 \mathrm{~d})$ spectra and a non-uniformly sampled 4D HCCH-TOCSY (800 MHz, $5 \mathrm{~d}$ ). Acquisition details of these spectra are similar to previously reported values (Stanek et al. 2016). All spectra were processed with Bruker Topspin and nmrPipe (Delaglio et al. 1995). Automatic peak-picking was performed in CcpNmr Analysis 2.4 (Vranken et al. 2005). Peak-lists were exported in Xeasy format for analysis with FLYA (Schmidt and Güntert 2012). The output was used as a guideline for manual spin-system linking and site-specific resonance assignment in CcpNmr. 
Results

Crystallization is the most commonly used and most successful method to perform solid-state NMR experiments on a soluble protein. While X-ray diffraction requires large single crystals, this is not a requisite for solid-state NMR. Since considerable sample amounts to fill an NMR rotor are required, crystal quantity often takes precedence over quality. Traditional sample preparation protocols involve batch methods to produce tens of milligrams of microcrystals that are required to fill a large NMR rotor $(3.2$ or $4 \mathrm{~mm})$ (Böckmann et al. 2003; Martin and Zilm 2003; Schmidt et al. 2007). The development of small sized rotors $(0.5-1.3 \mathrm{~mm})$ reduces by an order of magnitude the quantities required, allowing the direct exploitation of traditional vapor diffusion crystallization methods to produce the NMR samples.

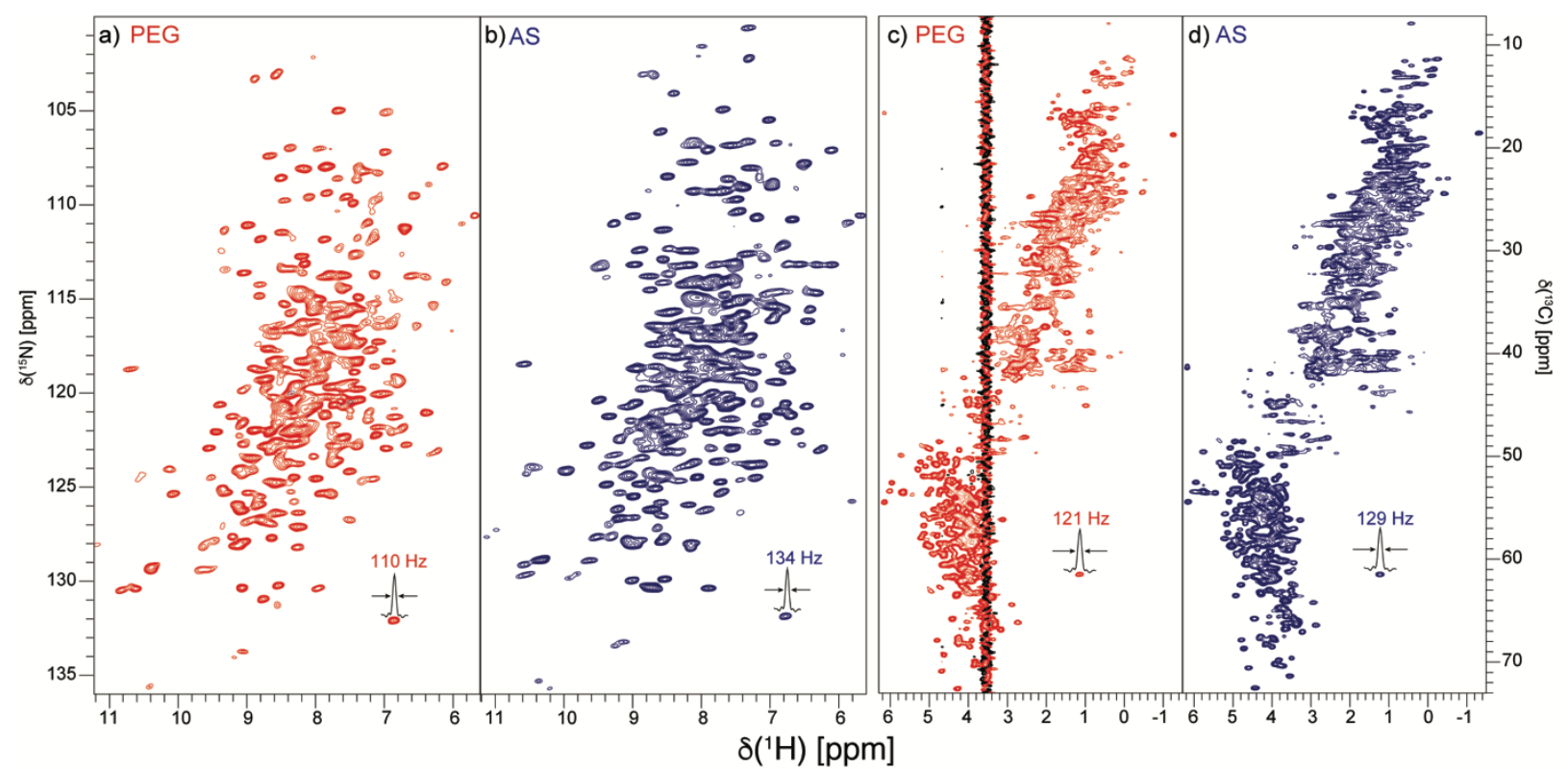

Figure 1: $(\mathrm{H}) \mathrm{NH}$ spectrum of ${ }^{13} \mathrm{C},{ }^{15} \mathrm{~N}$ MBP PEG (a) and AS crystals (b). (H)CH spectrum of the PEG (c) and AS sample (d). Both spectra were recorded at $B_{0}=23.5 \mathrm{~T}\left(\omega\left({ }^{1} \mathrm{H}\right) / 2 \pi=1 \mathrm{GHz}\right)$, the PEG sample was measured at $111 \mathrm{kHz} \mathrm{MAS}$ and $\mathrm{T}(\mathrm{read})=250 \mathrm{~K}$, the AS sample at $107 \mathrm{kHz}$ MAS and $\mathrm{T}(\mathrm{read})=260 \mathrm{~K}$.

Crystallization of MBP for NMR was initially screened around established conditions using polyethylene glycol (PEG) as precipitant (Spurlino et al. 1991; Sharff et al. 1992; Sharff et al. 1993). Crystals were obtained in several conditions and production simply scaled up to larger drops and multiple wells. A single 24 well plate with $40 \mu \mathrm{l}$ sitting drops provides sufficient material to fill a $1.3 \mathrm{~mm}$ rotor. The first PEG sample resulted in $(\mathrm{H}) \mathrm{NH}$ spectra of high quality (Figure 1a), with large peak dispersion, witnessing a native wellfolded state of the protein. We observed an average proton linewidth of $110 \mathrm{~Hz}$, a value that approaches that of reference microcrystalline proteins such as GB1 at similar experimental conditions (Cala-De Paepe et al. 2017). Fully protonated samples offer direct access to side-chain information with aliphatic (H)CH 2D correlations (Stanek et al. 2016). The (H)CH spectrum of the PEG MBP sample (Figure 1c) shows indeed high resolution with several isolated CA-HA and methyl group peaks, but the high concentration of PEG introduces a large signal at $3.5 \mathrm{ppm}$, arising from naturally abundant ${ }^{1} \mathrm{H}-{ }^{13} \mathrm{C}$ groups in $\mathrm{PEG}$, hampering severely the application of HA-detected methods for resonance assignment. This signal could be removed either by using deuterated PEG (expensive) or by finding a different precipitation agent. We found ammonium sulfate (AS) as a suitable precipitant of MBP having the advantage of not containing any naturally abundant ${ }^{13} \mathrm{C}$ atoms that could introduce background signals into $(\mathrm{H}) \mathrm{CH}$-based detection schemes. The AS samples of MBP also resulted in very well resolved spectra with only a marginal increase in the average amide ${ }^{1} \mathrm{H}$ linewidth and the same linewidth of alpha ${ }^{1} \mathrm{H}$ resonances within experimental error. The undisputed advantage of this sample is the clean $(\mathrm{H}) \mathrm{CH}$ spectrum, providing full access to the CA-HA 
region and allowing full use of HA-detected NMR spectra of higher dimensionality. The high number of isolated peaks in $2 \mathrm{D}$ correlations makes good prospects for the unambiguous detection of distance restraints or for the measurement of site-specific dynamics.

To further characterize the fully protonated AS MBP sample, we evaluated bulk refocused coherence lifetimes ( $\left.\mathrm{T}_{2}{ }^{\prime}\right)$ at $107 \mathrm{kHz}$ MAS frequency on a $1 \mathrm{GHz}$ spectrometer. We obtained $\mathrm{T}_{2}$ ' of $3 \mathrm{~ms}$ for amide ${ }^{1} \mathrm{H}$, and 84,48 and $58 \mathrm{~ms}$, respectively for ${ }^{15} \mathrm{~N},{ }^{13} \mathrm{CA}$ and ${ }^{13} \mathrm{CO}$, consistently with other microcrystalline preparations at these conditions. The high sensitivity and the relatively long $\mathrm{T}_{2}$ ' lifetimes allowed the efficient acquisition of the more demanding triple resonance experiments which employ INEPT-type ${ }^{13} \mathrm{C}-{ }^{13} \mathrm{C}$ coherence transfers. On a side note, a significantly longer ${ }^{15} \mathrm{~N} \mathrm{~T}_{2}$ ' was observed at lower static field (110 $\mathrm{ms}$ at $800 \mathrm{MHz}$ and $195 \mathrm{~ms}$ at $500 \mathrm{MHz}$ ), suggesting the presence of residual motional processes contributing via a chemical shift anisotropy relaxation mechanism.

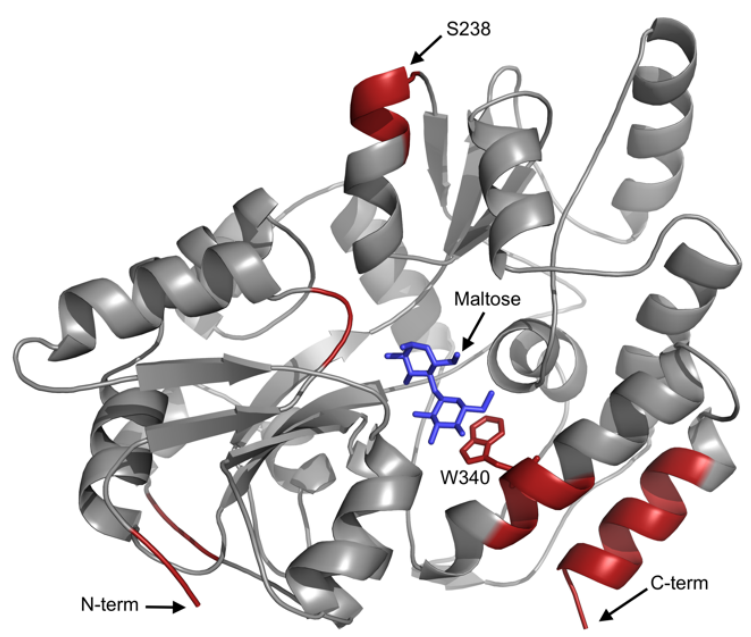

Figure 2: Visualization of the assignment completeness on the crystal structure (PDB 1ANF). Assigned residues are shown in grey, unassigned residues are highlighted in red. The ligand maltose is shown in blue. This figure was prepared with PyMOL (pymol.org).

Our starting point for backbone resonance assignment was the $82 \%$-complete result by the FLYA program based on the six simultaneously acquired amide- and alpha-proton detected triple resonance spectra of the RAVASSA approach, supplemented with two intra-residue correlation spectra reporting on CB chemical shift, ( $\mathrm{HCA}) \mathrm{CBCAHA}$ and $(\mathrm{HCA}) \mathrm{CB}(\mathrm{CA}) \mathrm{NH}$. Manual inspection and the addition of spectra correlating to ${ }^{13} \mathrm{CO}$ spins, namely $(\mathrm{H}) \mathrm{CONH},(\mathrm{H}) \mathrm{CO}(\mathrm{CA}) \mathrm{NH},(\mathrm{H}) \mathrm{COCAHA}$ and $(\mathrm{H}) \mathrm{CO}(\mathrm{N}) \mathrm{CAHA}$ allowed extension of the backbone assignment to 332 residues (90\%). First of all, the new experiments provide access to the previously unassigned $\mathrm{CO}$ chemical shifts, which are important for establishing the backbone dihedral angles. Second, the new data reduce the ambiguity of the sequential connections, improving the reliability of the results in the regions where the confidence of the RAVASSA output is lower due to reduced signal intensities, in turn increasing the number of residues for which backbone assignment was possible. Additionally, a 4D HCCH-TOCSY experiment allowed the assignment of several HB resonances. Few resonances observed in triple-resonance spectra remained unassigned, since no unambiguous connections could be found for these spin systems. The unassigned residues are highlighted on the crystal structure of MBP (PDB 1ANF) in Figure 2. Some loop regions remained unassigned including the flexible helical boundary around Serine 238, which is most likely due to unfavorable amplitude of dynamics at timescales comparable to the dipolar interactions and the inability to detect these resonances in experiments based on cross-polarization. The vastly unassigned C-terminal helix is solvent exposed and probably exhibits only minimal interaction with the globular core of the protein. This freedom might allow conformational exchange motions in this helix resulting in line broadening beyond the detection limit of NMR. More difficult to explain is the absence of resonances of Tryptophan 340 and neighboring residues, which are located in the middle of an alpha helix. There is no obvious reason for these residues to be mobile 
or disordered and our inability to assign could stem from spectral overlap and ambiguity. A more speculative explanation could be ligand induced static or dynamic conformational heterogeneity. In the crystal structure of holo-MBP, the sidechain of Trp-340 is oriented towards the maltose ligand (Figure 2). Conformational freedom of the bound ligand might induce slight variations in the side-chain orientation of Trp-340, resulting in heterogeneous broadening of its NMR resonances.

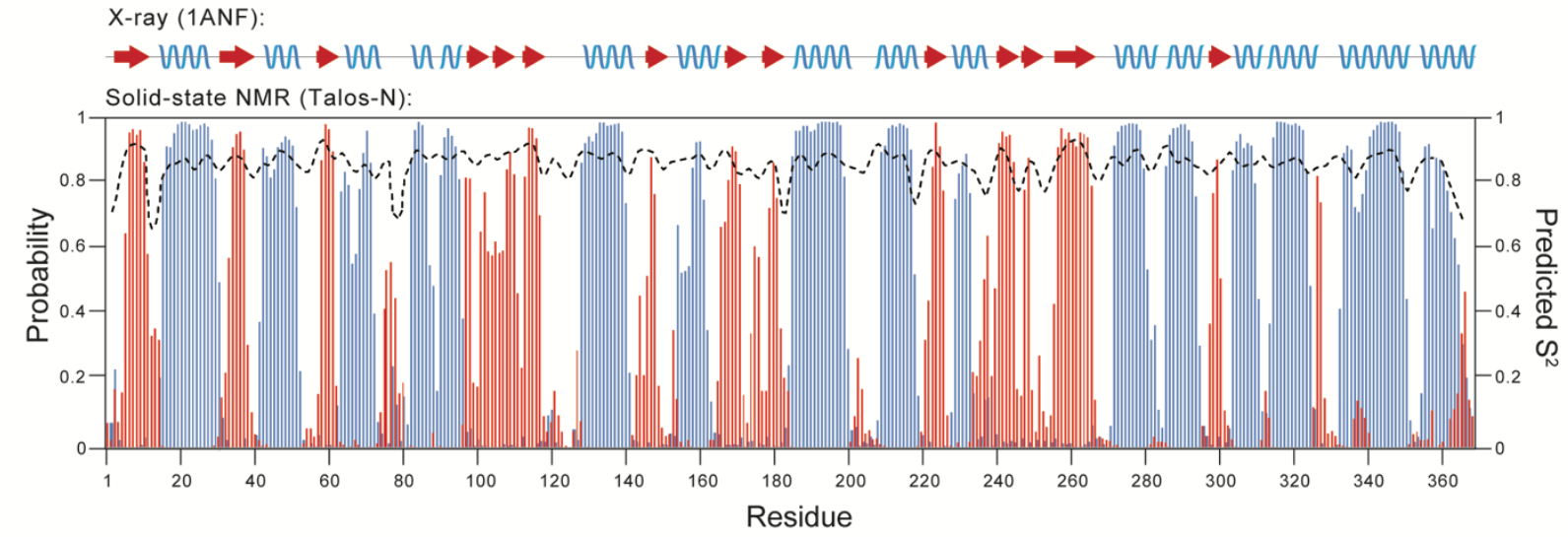

Figure 3: Secondary structure prediction from the assigned chemical shifts using TALOS-N. Beta-sheet and alpha-helical probability is plotted with red and blue bars, respectively. Predicted order parameters are shown with a dashed line. The secondary structure elements as present in the X-ray structural model (1ANF) are represented as red arrows and blue springs in the top panel.

The nearly complete assignment of all backbone $(\mathrm{H}, \mathrm{HA}, \mathrm{N}, \mathrm{CA}, \mathrm{CO})$ and $\mathrm{CB}$ resonances allowed a reliable secondary structure prediction with TALOS. The predicted secondary structure is generally in an excellent agreement with the X-ray structure (Figure 3). Small deviation worth mentioning are the predicted betastrands around residues 75 and 330 which are not present in the X-ray structure and might be of transient nature.

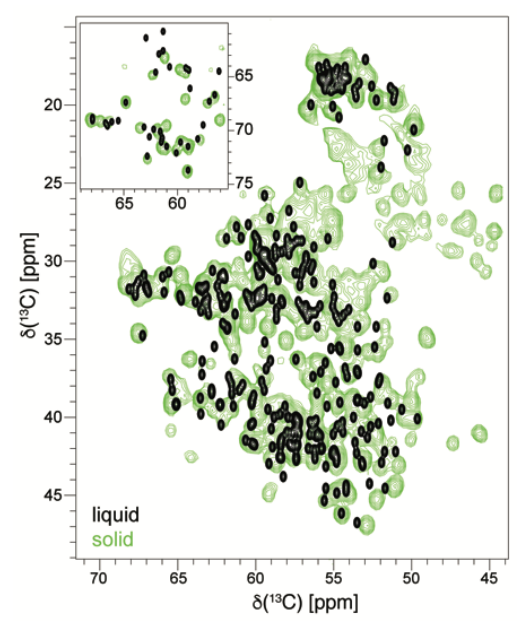

Figure 4: Comparison of solution and solid-state ${ }^{13} \mathrm{C}$ chemical shifts. A synthetic CBCA correlation (black contours) based on the solution NMR assignment of MBP in presence of maltotriose (BMRB 4987) is overlaid on a 2D CBCA projection of the 3D solid-state CBCAHA spectrum recorded with $107 \mathrm{kHz}$ MAS (green contours). The inset presents the characteristic region threonine and serine CA-CB correlations. Additional peaks in the CBCAHA spectrum arise from side chain ${ }^{1} \mathrm{H}-{ }^{13} \mathrm{C}$ correlations that cannot be easily suppressed in the experiment.

It is also interesting to compare the assignment with those previously obtained in solution NMR. However, ${ }^{1} \mathrm{H}$ and ${ }^{15} \mathrm{~N}$ chemical shifts are very sensitive to sample conditions such as $\mathrm{pH}$, temperature, ionic strength and type of ligand, and it is inconvenient to interpret ${ }^{15} \mathrm{~N}-{ }^{1} \mathrm{H}$ fingerprint $2 \mathrm{D}$ correlations in the solid on the basis of the assignments obtained from liquid preparations, for which the sample conditions differ 
significantly. The $\mathrm{CA}$ and $\mathrm{CB}$ chemical shifts on the other hand are more characteristic of protein secondary structure. Figure 4 shows a comparison between the $2 \mathrm{D}$ CBCA projection of the 3D solid-state CBCAHA spectrum with a synthetic CBCA spectrum created from the solution NMR assignment of MBP bound to a similar ligand, maltotriose (BMRB 4987). The spectra are in a very good agreement demonstrating a conserved secondary structure between the solution sample and the microcrystalline AS preparation.

In conclusion, we found sample conditions and obtained high quality ${ }^{1} \mathrm{H}$-detected NMR data under fast magic-angle spinning in terms of resolution and sensitivity. We demonstrated that the AS microcrystalline sample preserves the protein structure previously determined in single-crystals and in solution. The presented assignment constitutes the basis for further investigations of MBP dynamics and more in general for the development of new solid-state NMR methods for structure and dynamics of fully protonated proteins.

\section{Acknowledgements}

The expression vector was a gift by Prof. Gottfried Otting (ANU). The work was funded by the European Research Council (ERC) under the European Union's Horizon 2020 research and innovation program (ERC-2015-CoG GA 648974). J.S. was supported by the European Commission's REA with a MSCA fellowship (GA 661799).

\section{Accession Number}

${ }^{1} \mathrm{H},{ }^{13} \mathrm{C}$ and ${ }^{15} \mathrm{~N}$ backbone and side-chain chemical shifts of crystalline MBP have been deposited in the BioMagResBank (https://www.bmrb.wisc.edu) under the accession number 50089.

\section{Bibliography}

Böckmann A, Lange A, Galinier A, Luca S, Giraud N, Juy M, Heise H, Montserret R, Penin F, Baldus M (2003) Solid state NMR sequential resonance assignments and conformational analysis of the $2 \times$ $10.4 \mathrm{kDa}$ dimeric form of the Bacillus subtilis protein Crh. J. Biomol. NMR 27:323-339

Bonaccorsi M, Knight MJ, Le Marchand T, Dannatt HRW, Schubeis T, Salmon L, Felli IC, Emsley L, Pierattelli R, Pintacuda G (2020) Multimodal Response to Copper Binding in Superoxide Dismutase Dynamics. J Am Chem Soc 142:19660-19667 . https://doi.org/10.1021/jacs.0c09242

Boos W, Shuman H (1998) Maltose/Maltodextrin System of Escherichia coli: Transport, Metabolism, and Regulation. Microbiol Mol Biol Rev 62:204-229 . https://doi.org/10.1128/mmbr.62.1.204-229.1998

Cala-De Paepe D, Stanek J, Jaudzems K, Tars K, Andreas LB, Pintacuda G (2017) Is protein deuteration beneficial for proton detected solid-state NMR at and above $100 \mathrm{kHz}$ magic-angle spinning? Solid State Nucl Magn Reson 87:126-136 . https://doi.org/10.1016/j.ssnmr.2017.07.004

Delaglio F, Grzesiek S, Vuister G, Zhu G, Pfeifer J, Bax A (1995) NMRPipe: A multidimensional spectral processing system based on UNIX pipes. J Biomol NMR 6:277-293 . https://doi.org/10.1007/BF00197809

Evenäs J, Tugarinov V, Skrynnikov NR, Goto NK, Muhandiram R, Kay LE (2001) Ligand-induced structural changes to maltodextrin-binding protein as studied by solution NMR spectroscopy. J Mol Biol 309:961-974 . https://doi.org/10.1006/jmbi.2001.4695

Gardner KH (1998) Solution NMR studies of a 42 KDa Escherichia coli maltose binding protein/ $\beta$ cyclodextrin complex: Chemical shift assignments and analysis. J Am Chem Soc 120:11738-11748 . https://doi.org/10.1021/ja982019w

Madl T, Bermel W, Zangger K (2009) Use of Relaxation Enhancements in a Paramagnetic Environment for the Structure Determination of Proteins Using NMR Spectroscopy. Angew Chemie Int Ed 48:8259-8262 . https://doi.org/10.1002/anie.200902561

Mandala VS, Hong M (2019) High-sensitivity protein solid-state NMR spectroscopy. Curr. Opin. Struct. Biol. 58:183-190

Martin RW, Zilm KW (2003) Preparation of protein nanocrystals and their characterization by solid state NMR. J Magn Reson 165:162-174 . https://doi.org/10.1016/S1090-7807(03)00253-2

Rovó P, Smith CA, Gauto D, De Groot BL, Schanda P, Linser R (2019) Mechanistic Insights into 
Microsecond Time-Scale Motion of Solid Proteins Using Complementary $15 \mathrm{~N}$ and $1 \mathrm{H}$ Relaxation Dispersion Techniques. J Am Chem Soc 141:858-869 . https://doi.org/10.1021/jacs.8b09258

Schmidt E, Güntert P (2012) A new algorithm for reliable and general NMR resonance assignment. J Am Chem Soc 134:12817-12829 . https://doi.org/10.1021/ja305091n

Schmidt HLF, Sperling LJ, Gao YG, Wylie BJ, Boettcher JM, Wilson SR, Rienstra CM (2007) Crystal polymorphism of protein GB1 examined by solid-state NMR spectroscopy and X-ray diffraction. J Phys Chem B 111:14362-14369 . https://doi.org/10.1021/jp075531p

Selmke B, Borbat PP, Nickolaus C, Varadarajan R, Freed JH, Trommer WE (2018) Open and Closed Form of Maltose Binding Protein in Its Native and Molten Globule State As Studied by Electron Paramagnetic Resonance Spectroscopy. Biochemistry 57:5507-5512 . https://doi.org/10.1021/acs. biochem.8b00322

Seo MH, Park J, Kim E, Hohng S, Kim HS (2014) Protein conformational dynamics dictate the binding affinity for a ligand. Nat Commun 5: . https://doi.org/10.1038/ncomms4724

Sharff AJ, Quiocho FA, Rodseth LE, Quiocho FA (1993) Refined 1.8- $\AA$ Structure Reveals the Mode of Binding of $\beta$-Cyclodextrin to the Maltodextrin Binding Protein. Biochemistry 32:10553-10559 . https://doi.org/10.1021/bi00091a004

Sharff AJ, Rodseth LE, Spurlino JC, Quiocho FA (1992) Crystallographic Evidence of a Large LigandInduced Hinge-Twist Motion between the Two Domains of the Maltodextrin Binding Protein Involved in Active Transport and Chemotaxis. Biochemistry 31:10657-10663 . https://doi.org/10.1021/bi00159a003

Spurlino JC, Lu GY, Quiocho FA (1991) The 2.3-A resolution structure of the maltose- or maltodextrinbinding protein, a primary receptor of bacterial active transport and chemotaxis. J Biol Chem 266:5202-5219. https://doi.org/10.1016/s0021-9258(19)67774-4

Stanek J, Andreas LB, Jaudzems K, Cala D, Lalli D, Bertarello A, Schubeis T, Akopjana I, Kotelovica S, Tars K, Pica A, Leone S, Picone D, Xu Z-Q, Dixon NE, Martinez D, Berbon M, El Mammeri N, Noubhani A, Saupe S, Habenstein B, Loquet A, Pintacuda G (2016) NMR Spectroscopic Assignment of Backbone and Side-Chain Protons in Fully Protonated Proteins: Microcrystals, Sedimented Assemblies, and Amyloid Fibrils. Angew Chemie Int Ed 55:15504-15509 . https://doi.org/10.1002/anie.201607084

Stanek J, Schubeis T, Paluch P, Güntert P, Andreas LB, Pintacuda G (2020) Automated Backbone NMR Resonance Assignment of Large Proteins Using Redundant Linking from a Single Simultaneous Acquisition. J Am Chem Soc 142:5793-5799 . https://doi.org/10.1021/jacs.0c00251

Takeuchi K, Arthanari H, Shimada I, Wagner G (2015) Nitrogen detected TROSY at high field yields high resolution and sensitivity for protein NMR. J Biomol NMR 63:323-331 . https://doi.org/10.1007/s10858-015-9991-y

Tang C, Schwieters CD, Clore GM (2007) Open-to-closed transition in apo maltose-binding protein observed by paramagnetic NMR. Nature 449:1078-1082 . https://doi.org/10.1038/nature06232

Vranken WF, Boucher W, Stevens TJ, Fogh RH, Pajon A, Llinas M, Ulrich EL, Markley JL, Ionides J, Laue ED (2005) The CCPN data model for NMR spectroscopy: Development of a software pipeline. Proteins Struct Funct Genet 59:687-696 . https://doi.org/10.1002/prot.20449

Waugh DS (2016) Crystal structures of MBP fusion proteins. Protein Sci. 25:559-571

Yang D, Kay LE (1999) Improved 1HN-detected triple resonance TROSY-based experiments. J Biomol NMR 13:3-10 . https://doi.org/10.1023/A:1008329230975 